\title{
Placenta Prévia: Fatores de Risco para o Acretismo
}

\author{
Placenta Previa: Risk Factors for Accretion
}

Maria Regina Torloni, Antonio Fernandes Moron, Luiz Camano

\begin{abstract}
RESUMO
Objetivo: investigação dos fatores de risco associados ao acretismo em pacientes com placenta prévia $(P P)$.

Métodos: foi realizada uma análise retrospectiva caso-controle de todos os prontuários de pacientes com diagnóstico de PP que tiveram seus partos na Maternidade Escola Vila Nova Cachoeirinha (São Paulo) entre 1986 e 1998. O grupo com acretismo foi comparado ao grupo sem acretismo quanto à idade, paridade, antecedentes de abortamentos, curetagens $e$ cesarianas, o tipo de PP e a área predominante de inserção placentária. Para avaliar a associação entre a variável dependente (acretismo placentário) e as variáveis independentes (caracteristicas maternas e placentária) foram utilizados o teste do $\chi^{2}$, análise univariada e multivariada, considerando-se significantes os valores de $p<0,05$.

Resultados: o levantamento de 245 casos de PP evidenciou que os fatores de risco, significativamente associados ao acretismo, foram o tipo de PP centro-total (odds ratio (OR): 2,93) e o antecedente de duas ou mais cesarianas (OR: 2,54). Utilizando estes resultados foi possivel construir um modelo preditivo para a probabilidade de ter acretismo, segundo o qual uma paciente com PP centro-total e duas ou mais cesáreas teria uma probabilidade de 44,4\% de apresentar acretismo placentário.

Conclusões: os resultados deste estudo podem ajudar o obstetra a classificar suas gestantes com PP em diferentes categorias de risco para o acretismo, o que possibilita a adoção de medidas apropriadas para lidar com possiveis complicações no parto daquelas pacientes com maior risco de acretismo.
\end{abstract}

PALAVRAS-CHAVE: Placenta. Placenta prévia. Placenta acreta. Histerectomia.

Introdução

A placenta prévia (PP) vem se tornando cada vez mais freqüente, paralelamente ao crescente indice de cesarianas, um dos seus principais fatores predisponentes ${ }^{1,2}$. Uma das mais temidas complicações da PP é o acretismo placentário, caracterizado pela invasão excessiva do trofoblasto no miométrio, resultando em hemorragia significativa quando o obstetra tenta efetuar a dequitação. Diante desse diagnóstico, além da hemotransfusão serão necessárias manobras que vão desde curagem e curetagem até a histerectomia, conforme a extensão e o grau do acretismo

Departamento de Obstetrícia

Universidade Federal de São Paulo, Escola Paulista de

Medicina e Maternidade Escola de Vila Nova Cachoeirinha

- São Paulo, SP

Correspondência: Maria Regina Torloni

Rua Fagundes Dias, 172 apto. 31

04055-000 - São Paulo - SP placentário. Na última década o acretismo placentário superou a atonia uterina como principal causa de histerectomia por hemorragia pósparto $^{3}$.

O diagnóstico anteparto do acretismo na PP continua desafiando os obstetras. Na ultrasonografia (USG) os sinais de acretismo são sutis e a visibilização de toda a interface placentamiométrio é freqüentemente dificil, sobretudo nas PP de inserção predominantemente posterior e nas centrais ${ }^{4}$. Em alguns casos, a dopplervelocimetria colorida ${ }^{5}$ permite a suspeita de acretismo, porém o custo do equipamento ainda limita sua ampla utilização, assim como o da ressonância magnética, também testada com sucesso neste diagnóstico ${ }^{5}$. Como a maioria das maternidades brasileiras não dispõe de equipamentos sofisticados, na prática o diagnóstico de acretismo na PP continua sendo feito quase sempre no momento do parto, de forma clínica, sendo posteriormente 
confirmado pelo patologista naqueles casos submetidos à histerectomia.

Infelizmente, a placenta prévia acreta (PPA) vem se tornando cada vez mais freqüente. Em 1952, de cada 1.000 gestantes com PP, apenas 2 tinham acretismo associado ${ }^{6}$. Em 1980 a cifra elevou-se 40,5 / $1.000^{7}$, em 1985 para $99 / 1.000^{8}$ e a partir de 1995 já ultrapassava os $150 / 1.000^{9-11}$. Enquanto alguns atribuem esta tendência ao número crescente de gestantes com PP e idade avançada ou com antecedentes de abortamento e curetagem, outros apontam a cesariana prévia (cada vez mais freqüente) como principal fator predisponente para o acretismo. É provável que existam múltiplas variáveis maternas e placentárias envolvidas, interagindo entre si e predispondo ao acretismo na PP, e que algumas pacientes, em decorrência de características específicas, teriam um maior risco para desenvolver esta associação.

Ao realizar o parto de uma gestante com PPA o obstetra estará diante de uma situação de extremo risco para hemorragias, e o resultado final dependerá da sua aptidão em fazer o diagnóstico correto do acretismo, da sua experiência e habilidade técnica em realizar os procedimentos cirúrgicos indicados, da disponibilidade imediata de volumes adequados de sangue para transfusão e da competência dos anestesistas e intensivistas em manter as condições hemodinâmicas da paciente durante e após a cirurgia. Se o obstetra pudesse prever, de forma clínica e sem recursos tecnológicos sofisticados, qual a gestante com PP mais propensa ao acretismo, poderia preparar uma série de recursos capazes de garantir uma melhor assistência para esta paciente na hora do parto, reduzindo os seus riscos e até evitando a sua morte.

O objetivo deste trabalho foi o estudo dos fatores predisponentes ao acretismo na PP, avaliando o seu risco relativo.

\section{Pacientes e Métodos}

O estudo foi do tipo caso-controle, sendo analisados retrospectivamente todos os prontuários das pacientes com diagnóstico de PP que tiveram seus partos (vaginal ou cesáreo) na Maternidade Escola de Vila Nova Cachoeirinha (MEVNC) entre 1986 e 1998. Situada na periferia da zona norte de São Paulo, a MEVNC é uma instituição pública municipal especializada em gestações de alto risco que atende pacientes de baixo nivel socioeconômico encaminhadas de toda a cidade e de alguns municipios vizinhos.
Foram selecionados apenas os casos com conceptos com peso superior a 499 g, vivos ou mortos. O diagnóstico de PP foi baseado no quadro clínico clássico (sangramento indolor, imotivado e recorrente a partir da 20 $0^{\mathrm{a}}$ semana) acompanhado de USG típica (borda placentária situada nas imediações ou recobrindo o orifício interno do colo), realizada até 2 semanas antes do parto por médicos do setor de imagem da MEVNC. Todos os tipos de PP foram aceitos (lateral, marginal, centro-parcial e centro-total). Nas pacientes submetidas à cesariana o diagnóstico foi confirmado na descrição da cirurgia.

Também foram incluídos os casos com clínica típica e sem USG realizada na MEVNC, porém com descrição de $\mathrm{PP}$ na cesariana. O local predominante da inserção placentária foi considerado "anterior" apenas nos prontuários em que havia descrição (ou foto) de USG apontando este achado ou naqueles casos em que a descrição da cesárea citava a existência de cotilédones nesta face. Os casos que não preencheram estes critérios foram classificados como "não-anterior".

Os casos foram subdivididos em dois grupos denominados PP e PPA, respectivamente sem e com acretismo associado. No grupo PPA foram incluídas tanto as pacientes com acretismo clinico como aquelas com acretismo histologicamente confirmado. O diagnóstico de acretismo clínico fundamentou-se na descrição da ausência focal, parcial ou total, de plano de clivagem entre a placenta e o útero, associada à hemorragia e/ou à necessidade de manobras não-habituais no atendimento ao secundamento (extração manual de fragmentos placentários, curetagem e/ou capitonagem). O diagnóstico histológico do acretismo baseou-se no laudo anatomopatológico das pacientes submetidas a histerectomia e incluiu todos os seus graus tanto em profundidade (acreta, increta ou percreta) como em extensão superficial (total, parcial ou focal).

Foram obtidas variáveis maternas (idade, paridade, número de abortamentos, curetagens e cesáreas) e placentárias (tipo e área de inserção predominante) e os dados foram armazenados em banco de dados do programa Epi-Info versão 6.1 para posterior comparação entre os 2 grupos (PP e PPA). A associação entre a presença de acretismo (variável dependente) e as características maternas e placentárias (variáveis independentes) foi avaliada pelo teste do $\chi^{2}$ e da análise univariada. A análise conjunta dos fatores de risco foi feita por meio da análise multivariada. $\mathrm{O} p$ foi considerado significativo quando menor que $5 \%$. O trabalho foi aprovado pela Comissão de Ética da instituição. 


\section{Resultados}

No período do estudo ocorreram 245 partos que preencheram os critérios de inclusão quanto à presença de $\mathrm{PP}$. O índice de acretismo nesta população foi de 19,2\% (47/245). Devido ao acretismo, 17 pacientes $(6,9 \%)$ foram submetidas a histerectomia (10 totais e 7 subtotais), o que possibilitou a comprovação histológica do diagnóstico. Em relação à sua extensão superficial ${ }^{12}$, todos esses casos foram classificados pelo patologista como parciais (apenas uma parte da placenta apresentava-se aderida ao miométrio). Quanto à profundidade $^{13}$, 15 eram do tipo acreta (invasão mínima do miométrio), uma increta (invadindo profundamente a camada muscular) e uma percreta (com envolvimento da serosa uterina anterior). As outras 30 pacientes (12,3\%) com acretismo foram tratadas de forma conservadora. Uma paciente incluída no grupo de acretismo clínico faleceu na cesárea em decorrência de choque hipovolêmico durante a tentativa de dequitação manual da placenta. Como a família não concordou com a necropsia, não foi possivel a confirmação histológica do acretismo descrito no relato de cirurgia, e a paciente foi incluída no grupo dos 30 casos com tratamento clínico. A taxa de mortalidade materna nesta população de PP foi, portanto, de 4:1.000 nascimentos $(1 / 245)$.

Não houve associação estatisticamente significativa entre a presença de acretismo e a idade, o antecedente de abortamentos, curetagens e a área de inserção. A Tabela 1 resume a distribuição das pacientes segundo a presença das variáveis significativamente associadas ao acretismo na PP, com a análise univariada. Observamos associação estatisticamente significativa entre a paridade e o acretismo, com predomínio de multiparas no grupo do acretismo (OR: 6). O antecedente de cesárea também demonstrou associação significativa com o acretismo, sendo que o risco de acretismo foi 3 vezes maior nas pacientes com duas ou mais cesáreas do que naquelas sem este antecedente. Outra associação significativa foi entre o tipo de placenta prévia e a presença do acretismo: $51,1 \%$ das pacientes com acretismo tinham placentas do tipo centro-total, contra $27,8 \%$ daquelas sem acretismo, o que representou um risco 3 vezes maior. Em ordem decrescente de importância, a análise univariada identificou os seguintes fatores de risco para o acretismo em pacientes com PP: paridade 1-2 (OR: 6,20; IC: 1,3339,72), paridade $\geq 3$ (OR: 5,49; IC: $1,14-35,96)$, duas ou mais cesáreas (OR: 3,22; IC: $1,22-8,46$ ) e PP do tipo centro-total (OR: 3,20; IC: 1,29-8,15).

Tabela 1 - Distribuição das pacientes (porcentagens), valores dos odds ratio (OR) e respectivos intervalos de confiança (IC) para as variáveis de risco para o acretismo na placenta previa (análise univariada).

\begin{tabular}{|c|c|c|c|c|c|}
\hline Variável & $\begin{array}{l}\text { Com acretismo \% } \\
\qquad(\mathrm{n}=47)\end{array}$ & $\begin{array}{l}\text { Sem acretismo \% } \\
\qquad(n=198)\end{array}$ & OR & IC $95 \%$ & $p$ \\
\hline \multicolumn{6}{|l|}{ Paridade } \\
\hline 0 & 4,3 & 20,7 & 1,00 & Referência & \\
\hline $1-2$ & 55,3 & 43,4 & 6,20 & $1,33-39,72$ & 0,014 \\
\hline $3 e+$ & 40,4 & 35,9 & 5,49 & $1,14-35,96$ & 0,029 \\
\hline \multicolumn{6}{|l|}{ Cesáreas } \\
\hline 0 & 53,2 & 71,2 & 1,00 & Referência & \\
\hline 1 & 25,5 & 19,7 & 1,78 & $0,77-4,12$ & NS \\
\hline $2 \mathrm{ou}+$ & 21,3 & 9,1 & 3,22 & $1,22-8,46$ & 0,015 \\
\hline \multicolumn{6}{|l|}{ Tipo de PP } \\
\hline marginal & 19,1 & 33,3 & 1,00 & Referência & \\
\hline lateral & 29,8 & 38,9 & 1,33 & $0,50-3,60$ & NS \\
\hline central & 51,1 & 27,8 & 3,20 & $1,28-8,15$ & 0,008 \\
\hline
\end{tabular}

A análise multivariada permitiu avaliar no conjunto o peso específico de cada variável independente (idade, paridade, abortamentos, curetagens, cesáreas, tipo de PP e área de inserção) sobre a ocorrência do acretismo (variável de- pendente), eliminando possiveis confusões entre algumas variáveis. Nesta análise, as variáveis independentes que se associaram significativamente com a ocorrência de acretismo na PP foram o antecedente de 2 ou mais cesáreas (OR: 
2,54; IC: $1,02-6,36)$ e o tipo central de placenta prévia (OR: 2,93; IC: 1,24-6,90) (Tabela 2). A partir destes resultados, pode-se definir uma função (modelo de regressão logística) para estimar a probabilidade de haver acretismo. Baseada nesta equação ficaram definidas as probabilidades de ocorrência de acretismo para os diversos grupos de pacientes (Tabela 3).

Tabela 2 - Valores dos odds ratio (OR) e respectivos intervalos de confiança (IC) para as variáveis de risco para o acretismo na placenta de inserção baixa (análise multivariada).

\begin{tabular}{cccl}
\hline Variável & OR ajustado & IC 95\% & p \\
\hline Cesárea & & & \\
0 & 1,00 & Referência & \\
1 & 1,68 & $0,77-3,70$ & NS \\
$\geq 2$ & 2,54 & $1,02-6,36$ & 0,046 \\
Tipo de PP & & & \\
$\quad$ marginal & 1,00 & Referência & \\
lateral & 1,41 & $0,57-3,50$ & NS \\
central & 2,93 & $1,24-6,90$ & 0,014 \\
\hline NS= não-significante & & &
\end{tabular}

Tabela 3 - Probabilidade de ocorrência de acretismo em porcentagem estimada a partir do modelo de regressão logística múltipla, segundo o tipo de PP e o antecedente de cesarianas.

\begin{tabular}{cccc}
\hline & \multicolumn{3}{c}{ Tipo de PP } \\
\hline Antecedente de cesariana & Marginal & Lateral & Central \\
0 & 9,7 & 15,3 & 21,5 \\
1 & 13,2 & 20,3 & 27,8 \\
$\geq 2$ & 23,9 & 34,6 & 44,4 \\
\hline
\end{tabular}

Conforme aponta a Tabela 3, a paciente com menor probabilidade de ter acretismo $(9,7 \%)$ seria aquela com placenta prévia do tipo marginal e nenhuma cesárea anterior, ao passo que a paciente com maior probabilidade de ter acretismo $(44,4 \%)$ seria aquela com placenta prévia centrototal e duas ou mais cesáreas anteriores.

\section{Discussão}

$\mathrm{O}$ acretismo foi freqüente neste estudo, acometendo $19,2 \%$ das pacientes com PP, o que equivale a quase um em cada cinco casos. Na literatura, os autores que também utilizaram o critério clínico para o diagnóstico de acretismo apontam taxas que variam de 1,2 até $23,2 \%^{8,10,14-17}$. Por ser subjetivo, o diagnóstico clínico de acretismo sofre a influência de diversas variáveis, incluindo a experiência do obstetra, o que pode explicar, em parte, a amplitude da variação observada entre os diversos estudos. Outra possivel explicação para a elevada incidência de acretismo deste estudo seria o grande contingente de pacientes em nossa população com antecedentes de cesáreas $(32,2 \%)$, variável consistentemente apontada como importante fator predisponente à excessiva invasão trofoblástica. Todos os trabalhos que mostraram incidência de acretismo inferior à nossa tinham também proporções menores de pacientes com cesarianas anteriores, variando de $10,2^{14}$ a $21,2 \%{ }^{17}$.

A incidência de acretismo histologicamente confirmado foi de $6,9 \%$, valor que se situa entre os $2,6-17,6 \%$ assinalados nos trabalhos que utilizaram este critério ${ }^{9,11,18,19}$. Os autores que observaram incidências de acretismo mais próximas das nossas (Miller et al. ${ }^{11}$ com 9,3\% e Nielsen et al. ${ }^{19}$ com $7,4 \%$ ) coincidentemente também foram os que tiveram as taxas mais semelhantes de pacientes com cesáreas anteriores $(31,5 \%$ e $30,9 \%$, respectivamente). $\mathrm{O}$ trabalho com maior incidência de acretismo histologicamente confirmado (Lira Plascencia ${ }^{9}$ com $17,6 \%$ ) foi também aquele com a maior porcentagem de pacientes com cesáreas anteriores (44,3\%). Portanto, independente do critério diagnóstico utilizado (clínico ou histológico), parece evidente que quanto maior a porcentagem de pacientes no trabalho com antecedente de cesárea, maior será a taxa de acretismo placentário encontrado pelo autor.

A paridade elevada figura para alguns $s^{9,20,21}$ como possivel fator predisponente ao acretismo. Esses autores justificam seus achados, apontando que os sucessivos partos e dequitações talvez venham a comprometer o mecanismo regenerativo do endométrio, ou simplesmente aumentem a probabilidade de exposição a fatores predisponentes ao acretismo, como traumas e infecções. Porém, nenhum desses trabalhos avaliou estatisticamente seus resultados. Estudos com análises estatísticas (univariadas) ${ }^{11,15,17}$ não observaram diferenças significativas na paridade das pacientes com e sem acretismo na PP. Observamos um aumento significativo na freqüência de acretismo com o aumento da paridade na análise univariada, todavia quando este parâmetro foi avaliado especificamente, sem o efeito das outras variáveis (análise multivariada), a paridade não mostrou ser fator de risco significativo para o acretismo. Isto pode ser atribuído ao efeito do número de cesáreas sobre a paridade; isto é, o fator de risco maior para o acretismo não seria a paridade elevada em si, mas o antecedente de cesárea. 
Apesar de vários autores apontarem o grande número de pacientes com antecedente de abortamentos no grupo de PPA, apenas Zaki et al. ${ }^{16}$ analisaram estatisticamente (com teste do $\chi^{2}$ ) esta variável, encontrando significância limítrofe em seus resultados. Miller et al. ${ }^{11}$ avaliaram o antecedente de curetagens como fator de risco e também não encontraram relação com a ocorrência de acretismo na PP. Em nosso estudo os antecedentes de abortos e curetagens foram igualmente freqüentes nos grupos de PP e PPA, não representando portanto um fator de risco específico para o acretismo.

As pacientes com placenta prévia freqüentemente têm cicatriz uterina, sendo este um dos fatores envolvidos na sua etiopatogenia. Ao compararmos os grupos com e sem acretismo, observamos diferença significativa na distribuição das pacientes quanto ao antecedente de cesáreas. $\mathrm{O}$ risco relativo para o acretismo foi 3 vezes maior para as pacientes com 2 ou mais cesáreas. Vários trabalhos também apontam a cesárea anterior como fator de risco para o acretismo na PP, concluindo que quanto maior o número de cirurgias, maior será este risco ${ }^{8-11,14,16,17}$. É provável que na presença de repetidas cicatrizes o endométrio do segmento inferior, naturalmente delgado, tenha maior dificuldade em sofrer o processo de decidualização, favorecendo cada vez mais o acretismo.

Em decorrência da histerorrafia situar-se sempre na parede uterina anterior, esperávamos que as placentas inseridas predominantemente nesta face tivessem uma maior freqüência de acretismo. Observamos um discreto predominio de acretismo nas placentas inseridas anteriormente $(46,8 \%)$, comparadas àquelas inseridas em outras áreas $(40,9 \%)$, porém sem significado estatístico. Miller et al. ${ }^{11}$ também analisaram este aspecto, contudo utilizaram um critério diferente, criando uma categoria "placenta recobrindo cicatriz de cesárea", que incluía tanto as de inserção anterior, como muitas do tipo centro-total sem outras especificações. Observaram 29\% de acretismo nesse grupo e 6,5\% nas demais localizações, sendo esta diferença significativa. Acreditamos que com uma casuística maior, ou se utilizássemos uma definição de inserção anterior menos rígida, teriamos possivelmente obtido resultados estatisticamente significativos.

Houve maior freqüência de acretismo na PP do tipo centro-total em relação às demais variedades, sendo esta diferença estatisticamente significativa. É possivel que a PP centro-total seja a variedade que encontre as piores condições de suprimento vascular e esta escassez nutricional ative mecanismos compensatórios que resultam na maior penetração vilositária. Nenhum dos trabalhos a que tivemos acesso analisou de forma específica e isolada esta variável como fator de risco para o acretismo.

O modelo de regressão logística evidenciou que a gestante com PP do tipo central e antecedente de duas ou mais cesáreas seria a de maior risco para o acretismo. Na prática, esta informação pode ser de grande utilidade para o obstetra, possibilitando uma serie de ações importantes para garantir o melhor resultado possivel. Em primeiro lugar, esta paciente e sua familia poderiam ser informados ainda durante o pré-natal deste risco e suas possiveis conseqüências (transfusão, histerectomia, complicações operatórias), evitando problemas futuros relacionados à morbidade e até mortalidade maternas inesperadas. O obstetra poderia também solicitar para esta gestante exames mais sofisticados (Doppler colorido, ressonância magnética $)^{5}$ na tentativa de confirmar o acretismo, apesar de não existir ainda na atualidade evidência suficiente para justificar a utilização rotineira destes métodos. Seria importante ainda programar este parto para que ocorra em hospital adequadamente equipado para lidar com emergências hemorrágicas (banco de sangue estocado, vaga na UTI, equipes anestésica e cirúrgica experientes).

A prevenção do acretismo nas pacientes com PP depende da compreensão exata dos mecanismos etiopatogênicos envolvidos. Como este conhecimento é ainda incompleto, resta-nos a atuação sobre os fatores de risco. Identificamos neste estudo duas variáveis que aumentam significativamente o risco de acretismo na PP. Como é atualmente impossivel atuar sobre o determinismo da implantação placentária, resta a tentativa de reduzir o uso indiscriminado de cesarianas, tão disseminado em nosso país. Na prevenção da PPA, seria portanto importante incentivar o parto vaginal nas pacientes com uma cesariana anterior e talvez encorajar o uso de anticoncepção definitiva naquelas com duas ou mais cesarianas.

Este trabalho procurou identificar os principais fatores maternos e placentários que aumentam a probabilidade de acretismo em pacientes com PP. Dentre as diversas variáveis de risco apontadas como relevantes pela literatura e por nós pesquisadas, pudemos concluir que o antecedente de duas ou mais cesáreas e a PP do tipo centro-total aumentam significativamente essa probabilidade. Ao assistir o parto dessas pacientes, os obstetras devem ficar alertas e preparados para a possibilidade de complicações hemorrágicas. 


\section{SUMMARY}

Purpose: to investigate risk factors associated with accretion in placenta previa (PP) patients.

Methods: this was a retrospective case-control study of all the records of patients who delivered between 1986-1998 at Maternidade Escola de Vila Nova Cachoeirinha (São Paulo) with a diagnosis of placenta previa. The groups with and without accretion were compared regarding age, parity, previous history of miscarriage, curettage and cesarean section, type of PP and predominant area of placental attachment. Possible associations between the dependent (accretion) and independent (maternal and placental characteristics) variables were evaluated using the $\chi^{2}$ test, univariate and multivariate analyses.

Results: reviewing 245 cases of PP, two risk factors were significantly associated with accretion: central placenta previa (odds ratio (OR): 2.93) and two or more previous cesarean sections(OR: 2.54). Based on these data, a predictive model was constructed, according to which a patient with central PP and two more previous cesarean sections has a $44.4 \%$ risk for accretion.

Conclusions: results of the current study may help obstetricians in the classification of their patients with PP in different risk categories for accretion. This could be useful in preparing for possible delivery complications in those patients considered at a higher risk for accretion.

KEY WORDS: Placenta. Placenta previa. Placenta accreta. Hysterectomy.

\section{Referências}

1. Ananth CV, Smulian JC, Vintzileos A. The association of placenta previa with the history of cesarean delivery and abortion: a meta-analysis. Am J Obstet Gynecol 1997; 177:1071-8.

2. Frederiksen MC, Glassenberg R, Stika CS. Placenta previa: a 22-year analysis. Am J Obstet Gynecol 1999; 180:1432-7.

3. Cunningham RG, Mac Donald FC, Gant NG, et al.. Williams Obstetrics. 20 ${ }^{\text {th }}$ ed. East Norwalk: Appleton \& Lange; 1997. p.761.

4. Finberg HJ, Williams JW. Placenta accreta: prospective sonographic diagnosis in patients with placenta previa and prior cesarean section. J Ultrasound Med 1992; 11:333-43.

5. Levine D, Hulka CA, Ludmir J, Li W, Edelman RR. Placenta accreta: evaluation with color Doppler US, power Doppler US and MR imaging. Radiology 1997; 205:773-6.
6. Kistner RW, Hertig AT, Reid DE. Simultaneously occurring placenta previa and placenta accreta. Surg Gynecol Obstet 1952; 94:141-51.

7. Read JA, Cotton DB, Miller FC. Placenta accreta: changing clinical aspects and outcome. Obstet Gynecol 1980; 56:31-4.

8. Clark SL, Koonings PP, Phelan JP. Placenta previa/ accreta and prior cesarean section. Obstet Gynecol 1985; 66:89-92.

9. Lira Plascencia J, Ibarguengoitia Ochoa F, Argueta Zúñiga M, Karchmerk S. Placenta previa/acreta y cesárea previa: experiencia de cinco años en el Instituto Nacional de Perinatología. Ginecol Obstet Mex 1995; 63:337-40.

10.Corrêa MAC, Orsatto Jr S, Torloni MR, Moron AF, Sass N. Influência da cesárea anterior sobre o acretismo em pacientes com placenta prévia. Rev Bras Ginecol Obstet 1997; 19:105-9.

11. Miller DA, Chollet JA, Goodwin TM. Clinical risk factors for placenta previa-placenta accreta. Am J Obstet Gynecol 1997; 177:210-4.

12.Aaberg ME, Reid DE. Manual removal of the placenta: a policy of treatment. Am J Obstet Gynecol 1945; 49:368-77.

13.Irving FC, Hertig AT. A study of placenta accreta. Surg Gynecol Obstet 1937; 64:178-200.

14.To WW, Leung WC. Placenta previa and previous cesarean section. Int $\mathrm{J}$ Gynaecol Obstet 1995; 51:25-31.

15.Gorodeski IG, Bahari CM, Holzinger M, Schachtzer A, Neri A. Placenta previa with focal accretion. Isr J Med Sci 1982; 18:277-80.

16.Zaki ZM, Bahar AM, Ali ME, Albar HA, Gerais MA. Risk factors and morbidity in patients with placenta previa accreta compared to placenta previa non-accreta. Acta Obstet Gynecol Scand 1998; 77:391-4.

17. Chattopadhyay SK, Kharif H, Sherbreeni MM. Placenta praevia and accreta after previous caesarean section. Eur J Obstet Gynecol Reprod Biol 1993; 52:151-6.

18.Piya-Anant M. Management of previous caesarean section presented with placenta previa. J Med Assoc Thai 1987; 70:673-7.

19.Nielsen TF, Hagberg H, Ljungblad U. Placenta previa and antepartum hemorrhage after previous cesarean section. Gynecol Obstet Invest 1989; 27:88-90.

20.Kitchen DH. Placenta accreta, percreta and praevia accreta. Aust N Z J Obstet Gynaecol 1978; 18:238-41.

21. Morison JE. Placenta accreta. A clinicopathologic review of 67 cases. Obstet Gynecol Annu 1978; 7:107-23. 